\title{
INDICADORES DE DEPRESSÃO EM IDOSOS INSTITUCIONALIZADOS
}

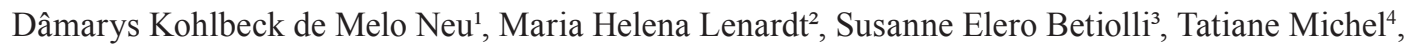
Mariluci Hautsch Willig ${ }^{4}$

\begin{abstract}
RESUMO: Trata-se de estudo quantitativo descritivo transversal, cujo objetivo foi identificar os indicadores de depressão em idosos residentes em uma Instituição de Longa Permanência para Idosos de Curitiba, Paraná. A instituição abriga 78 idosos, de ambos os sexos, com diferentes graus de dependência. A amostra foi constituída por 21 idosos após screening cognitivo e aplicação dos critérios de inclusão e exclusão. Os dados foram coletados por meio do questionário multidimensional - BOAS (Brazil Old Age Schedule) e analisados conforme estatística descritiva e distribuição de frequência. Do total de idosos, 57,14\% não apresentaram indicadores de depressão; 28,57\% possuíam depressão leve; e 14,29\% depressão severa. Infere-se que os idosos apresentavam elevado índice de sintomatologia depressiva, entretanto, não se refere ao diagnóstico de depressão. A detecção precoce dos sintomas ajuda a evitar o desenvolvimento dessa patologia e prevenir seus efeitos à saúde e qualidade de vida de idosos.
\end{abstract}

PALAVRAS-CHAVE: Instituição de longa permanência para idosos; Idoso; Depressão; Enfermagem.

\section{INDICATORS FOR DEPRESSION AMONG ELDERLY IN LONG-TERM CARE INSTITUTIONS}

ABSTRACT: A quantitative transversal descriptive study, whose objective was to identify indicators for depression among elderly residents in a Long-term Care Home for the Elderly in Curitiba, Paraná. The institution is home to 78 elderly residents of both sexes with differing degrees of dependency. The sample was made up of 21 elderly persons after cognitive screening and application of inclusion and exclusion criteria. Data was collected via a multidimensional questionnaire BOAS (Brazil Old Age Schedule) and analysed by descriptive statistics and frequency distribution. Of the sample, $57.14 \%$ presented no markers for depression; $28.57 \%$ had mild depression: and $14.29 \%$ had severe depression. It is inferred that they presented a higher level of depressive symptomatology, however, the diagnosis of depression is not referred to. Early detection of these symptoms helps to avoid the development of this pathology and prevent its effects on health and quality of life among the elderly.

KEYWORDS: Long-term care facilities; Elderly; Depression; Nursing.

\section{INDICADORES DE DEPRESIÓN EN ANCIANOS INSTITUCIONALIZADOS}

RESUMEN: Este es un estudio cuantitativo descriptivo transversal, cuyo objetivo fue identificar los indicadores de depresión en ancianos residentes en una Institución de Larga Permanencia para Ancianos de Curitiba, Paraná. La institución abriga 78 ancianos, de ambos sexos, con diferentes grados de dependencia. La muestra fue constituida por 21 ancianos despues de screening cognitivo y aplicación de los criterios de inclusión y exclusión. Los datos fueron recogidos por medio del cuestionario multidimensional - BOAS (Brazil Old Age Schedule) y analizados conforme estadística descriptiva y distribución de frecuencia. Del total de ancianos, 57,14\% no presentaron indicadores de depresión; 28,57\% poseían depresión leve; y 14,29\% depresión severa. Se deduce que los ancianos presentaban elevado índice de sintomatología depresiva acerca del diagnóstico de depresión. La detección precoz de los síntomas ayuda a evitar el desarrollo de esa patología y prevenir sus efectos a la salud y cualidad de vida de ancianos.

PALABRAS-CLAVE: Institución de larga permanencia para ancianos; Anciano; Depresión; Enfermería.

'Enfermeira. Mestranda pelo Programa de Pós-Graduação em Enfermagem da Universidade Federal do Paraná - PPGENF UFPR. Bolsista REUNI. Membro do Grupo Multiprofissional de Pesquisa sobre Idosos - GMPI.

${ }^{2}$ Enfermeira. Doutora em Filosofia da Enfermagem. Professora do PPGENF UFPR. Líder do GMPI.

${ }^{3}$ Enfermeira. Mestranda pelo PPGENF UFPR. Bolsista CAPES. Membro do GMPI.

${ }^{4}$ Enfermeira. Mestre em Enfermagem. Doutoranda pelo PPGENF UFPR. Membro do GMPI.

Autor correspondente:

Dâmarys Kohlbeck de Melo Neu

Universidade Federal do Paraná

Rua Adir Pedroso, 777 - 83065-110 - São José dos Pinhais-PR-Brasil

E-mail: damy_neu@yahoo.com.br
Recebido: 27/06/11

Aprovado: $12 / 08 / 11$

Cogitare Enferm. 2011 Jul/Set; 16(3):418-23 


\section{INTRODUÇÃO}

Os novos arranjos domiciliares e a redução da possibilidade de apoio e cuidado familiar aos idosos, entre outros fatores, têm contribuído para a ocorrência da fragilização do suporte familiar ao idoso. Para os casos de insuficiência familiar grave em que outras modalidades de atendimento não foram suficientes para garantir o cuidado, o internamento em instituição de longa permanência é considerado uma alternativa digna ${ }^{(1)}$.

No âmbito das instituições de longa permanência é imprescindível a avaliação psicológica, pois ela contribui para a identificação de alterações que podem sinalizar patologias associadas, bem como atentar para variações cognitivas e de humor, importantes na manutenção da qualidade de vida desses indivíduos.

A prevalência de depressão entre idosos varia conforme o local do estudo, entretanto, sabe-se que a ocorrência dessa patologia entre os institucionalizados é de 10 a $22 \%$ maior quando comparada aos idosos que vivem na comunidade ${ }^{(2)}$, e as taxas de sintomas depressivos variam de 10 a $30 \%{ }^{(3)}$.

Além dos fatores advindos do envelhecimento que podem levar à depressão nos idosos, a institucionalização também pode ser considerada um fator estressante e desencadeador dessa patologia. Os idosos residentes em asilos apresentam risco aumentado de desenvolver depressão, principalmente nos primeiros meses após a internação ${ }^{(4)}$.

Entre as diferentes abordagens relacionadas à depressão e ao idoso, encontra-se aquela referente à funcionalidade do indivíduo, na qual os sintomas depressivos são expressivamente severos a ponto de prejudicar a função social e física. Essa ainda considera fatores multidimensionais, como o envolvimento social e a satisfação com a vida ${ }^{(5)}$. Sua utilização foi pertinente neste trabalho, ao considerar que os indicadores de depressão nos idosos institucionalizados foram determinados por meio da utilização da seção de saúde mental de um questionário multidimensional específico para a avaliação do idoso.

No ambiente da Instituição de Longa Permanência para Idosos - ILPI, devido à proximidade ao idoso, a equipe de enfermagem tem maiores condições de manter-se atenta à presença de sintomas depressivos. A identificação destes é relevante ao se considerar que a depressão pode agravar ou desencadear condições clínicas desfavoráveis, além de interferir diretamente na capacidade funcional dos idosos e aumentar a demanda por cuidados, o que contribui negativamente para a qualidade de vida dos institucionalizados. Diante do exposto, o estudo teve como objetivo identificar os indicadores de depressão em idosos residentes em uma Instituição de Longa Permanência do Município de Curitiba-PR.

\section{METODOLOGIA}

Trata-se de estudo quantitativo descritivo com delineamento transversal, realizado em uma Instituição de Longa Permanência para Idosos sediada em CuritibaPR. A instituição é de caráter privado, onde residem 78 idosos, com diferentes graus de dependência funcional, déficits cognitivos e físicos.

A população do estudo foi composta pelos idosos residentes na ILPI. Realizou-se a leitura dos prontuários, observação e diálogo com os idosos e com a equipe de enfermagem, com o objetivo de realizar a triagem inicial daqueles com capacidade física e cognitiva para responder ao questionário multidimensional de saúde - Brazil Old Age Schedule (BOAS). Nessa fase foram excluídos 46 idosos: em 21 deles observou-se demência em estágio avançado, 10 comunicavam-se exclusivamente por idioma estrangeiro, seis apresentavam afasia, cinco estavam hospitalizados, dois estavam há menos de três meses na instituição e dois recusaram-se a participar do estudo.

A partir dessa etapa, foi aplicado o Miniexame do Estado Mental (MEEM) para definição da amostra. Foram considerados os pontos de corte, segundo a escolaridade $^{(6)}$, de 13 pontos para analfabetos, 18 para os de escolaridade baixa e média, e 26 pontos para aqueles com escolaridade alta. Dos 32 idosos que realizaram o MEEM, 9 não atingiram os pontos de corte conforme seu grau de escolaridade e duas idosas interromperam sua participação no estudo, na fase de aplicação do questionário BOAS. A amostra final constituiu-se de 21 idosos.

Foram considerados os seguintes critérios de inclusão: possuir idade igual ou superior a 60 anos; residir na ILPI há pelo menos três meses; ser física e cognitivamente capaz de responder ao questionário multidimensional de saúde - BOAS; obter pontuação no MEEM acima dos pontos de corte; assinar o termo de consentimento livre e esclarecido. Os critérios de exclusão foram: expressar o desejo de interromper a participação; apresentar expectativa de vida inferior a seis meses, devido a doenças terminais devidamente diagnosticadas e documentadas; e idosos transferidos para outra ILPI ou hospital. 
Os dados foram coletados no período de agosto a novembro de 2009 por intermédio da aplicação do MEEM $^{(7-8)}$ para screening cognitivo e de entrevistas junto aos idosos, com intuito de identificar os indicadores de depressão por meio da seção de saúde mental do questionário multidimensional BOAS.

O questionário $\mathrm{BOAS}^{(9)}$ é composto por 9 seções, num total de 113 questões. Deste questionário foram excluídas as questões que não contemplavam especificidades da população residente em ILPI. Algumas atividades, como cozinhar o próprio alimento, atender ao telefone e lavar suas roupas, não fazem parte das atividades rotineiras executadas pelos idosos deste estudo.

Realizou-se estudo-piloto com o intuito de verificar a adequação do instrumento de pesquisa. Para esse trabalho empregaram-se as respostas dos idosos às questões referentes aos indicadores de depressão, contempladas na seção de saúde mental do questionário eleito para o estudo. Essa seção compreende 36 questões, das quais 27 são referentes aos indicadores de depressão e 9 são consideradas na avaliação dos indicadores de demência.

A análise dos indicadores de depressão foi realizada considerando-se apenas 22 questões específicas da seção de saúde mental, para as quais existe correspondência em pontuação ${ }^{(9)}$. Cada resposta dada a essas questões recebe uma pontuação e, para obter-se o score final, somam-se as pontuações recebidas. $\mathrm{O}$ idoso que apresentar até 7 pontos é considerado sem indicadores de depressão, se pontuar entre 8 e 12 é classificado com depressão leve, e aquele com score final acima de 13 pontos apresenta depressão severa ${ }^{(10)}$.

Os dados coletados foram organizados por estatística descritiva e distribuição de frequência, empregando-se o programa Excell e Epi Info versão 6.04. Os resultados são apresentados em tabelas e linguagem descritiva. $\mathrm{O}$ projeto de pesquisa foi encaminhado ao Comitê de Ética em Pesquisa do Setor de Ciências da Saúde da Universidade Federal do Paraná, o qual recebeu parecer favorável, com registro CAAE: 2066.0.00.091-09.

\section{RESULTADOS}

Participaram do estudo 21 idosos residentes na ILPI, sendo que 12 idosos (57,14\%) não apresentam indicadores de depressão, seis idosos (28,57\%) apresentam depressão leve e três $(14,29 \%)$ depressão severa.

Apresenta-se, na tabela 1(p. 421), as respostas dos idosos para as questões de pontuação da seção de saúde mental do questionário BOAS.
Destaca-se na tabela 1 que $71,4 \%$ dos idosos estiveram preocupados no último mês, $66,7 \%$ sentiam-se mais lerdos, com menos energia, e 57,1\% não mencionaram expectativas para o futuro.

\section{DISCUSSÃO}

Verifica-se que $42,86 \%$ dos idosos residentes na ILPI apresentam indicadores de depressão. Esse resultado é semelhante ao obtido em estudo realizado em Pernambuco com idosos institucionalizados, o qual revelou que $51 \%$ apresentavam sintomatologia depressiva ${ }^{(11)}$. A mesma taxa de depressão em idosos institucionalizados foi observada no trabalho desenvolvido em um asilo do Município de Mossoró, Rio Grande do Norte ${ }^{(12)}$.

Em investigação cujo objetivo foi avaliar a frequência de sintomas depressivos e o desempenho cognitivo de idosos institucionalizados e não institucionalizados, evidenciou-se maiores taxas de sintomas depressivos entre os institucionalizados ${ }^{(13)}$. Da mesma forma, pesquisa realizada com 615 idosos em Helsinki-Finlândia constatou prevalência quatro vezes maior de Depressão Maior entre institucionalizados, em comparação aos que vivem na comunidade ${ }^{(14)}$. O mesmo estudo sugere ainda que a depressão é rara entre idosos saudáveis, e comum entre idosos institucionalizados, com incapacidades.

Em estudo realizado em uma ILPI que abriga apenas idosas, foram detectados indicadores de depressão em $35 \%$ das participantes do estudo. As autoras da pesquisa destacam que a identificação de novos casos e o monitoramento dos tratamentos devem ser realizados por meio de escala específica, visto que a presença de sintomas depressivos causa um dano adicional e está associada à maior morbidade e mortalidade ${ }^{(15)}$.

Destaca-se, na tabela 1, que as respostas mais frequentes entre os idosos foram referentes a estar preocupado no último mês $(71,4 \%)$, sentir-se mais lerdo ou com menos energia (66,7\%), e não mencionar expectativas para o futuro $(57,1 \%)$. Em investigação com 544 idosos da comunidade, foi observado que os eventos que afetam a descendência, ou que estão relacionados ao cuidado a outro adulto e ao próprio bem-estar psicológico, foram relacionados com a presença de depressão. Destaca-se que aspectos como a incontrolabilidade, as dificuldades em desempenhar tarefas de cuidado ou sentimentos de ameaça ao próprio bem-estar pessoal podem imprimir um quadro sintomático depressivo $^{(16)}$.

Verifica-se ainda que $28,6 \%$ dos idosos com indicadores de depressão apresentaram dificuldades para 
dormir devido à preocupação, ansiedade ou pensamentos depressivos. A insônia está relacionada à depressão e é um sintoma muito comum, mas não exclusivo, nos idosos depressivos ${ }^{(4)}$.

O sono de idosos difere do sono de um adulto jovem principalmente por uma tendência a haver maior número de interrupções e a uma discreta diminuição da profundidade do sono. Estudorealizado para determinar a prevalência de depressão maior em idosos acima de 80 anos e comparar os padrões de sono e a função cognitiva entre grupos controles e sujeitos com depressão, mostrou que os sujeitos sem diagnóstico de doença de humor tiveram os mesmos padrões de sono dos sujeitos deprimidos. As diferenças verificadas entre os dois grupos apontam que os sujeitos deprimidos cochilavam menos tempo, à tarde, e tendiam a se descrever como menos dispostos ao acordar pela manhã, quando comparados com os grupos controles ${ }^{(17)}$.

Tabela 1 - Número de idosos que pontuaram em cada questão da seção de saúde mental do questionário BOAS. Curitiba, 2011

\section{Respostas às questões de pontuação}

Sempre se sente solitário

Esteve preocupado durante o último mês

Preocupa-se em relação a quase tudo

Teve dificuldade para dormir devido à preocupação, ansiedade, depressão ou pensamento depressivo

Teve dor de cabeça uma ou mais vezes no mês passado

Não se alimentou bem no último mês por falta de apetite ou por estar deprimido/preocupado/nervoso

Sente que está ficando mais lerdo ou com menos energia

Sente-se mais lerdo ou com menos energia no período da manhã

No último mês tem estado com menos energia que de costume

Atualmente sente falta de energia

Sentiu-se mais irritado / zangado no último mês

Tem se sentido triste ou deprimido no último mês

Tem se sentido triste ou deprimido por um período maior que poucas horas

Sente-se mais triste ou deprimido no início do dia

Chorou ou sentiu vontade de chorar no último mês

No último mês sentiu que viver não valia a pena

Rejeita o suicídio

Apresenta pensamentos suicidas

Arrepende-se sobre a vida e culpa-se sobre isso, mas não pensa no assunto atualmente

Arrepende-se sobre a vida, culpa-se sobre isso e pensa bastante sobre o assunto

Não menciona expectativas para o futuro

As expectativas para o futuro são descritas negativamente

Atualmente, sente que perdeu o interesse ou a satisfação pelas coisas

Perda de interesse causada pela depressão/nervosismo

Sente-se infeliz, atualmente n (\%)

$6(28,6)$

$15(71,4)$

$8(38,1)$

$6(28,6)$

$14(66,7)$

$9(42,8)$

$6(28,6)$

$5(23,8)$

$6(28,6)$

$3(14,3)$ 
Na tabela 1, observa-se que $19 \%$ dos idosos apresentaram cefaleia no último mês. Esse sintoma somático pode estar relacionado à depressão em idosos, pois é comum que esses pacientes não relatem sentimento depressivo, e queixem-se apenas de dores no corpo e de fadiga generalizada ${ }^{(4)}$.

Destaca-se que $66,7 \%$ dos idosos sentiam que estavam se tornando mais lerdos ou com menos energia. Entre esses idosos, 19\% sentiam-se mais lentos no período da manhã, 33,3\% estavam com menos energia em relação à habitual, e 23,8\% sentiam falta de energia, atualmente. Essa sintomatologia está geralmente presente no quadro clínico de depressão em idosos, e pode estar associada às dores nas costas, cefaleia e vertigem. $\mathrm{O}$ idoso com redução da energia, frequentemente, sente-se também cansado, desanimado, fraco e sem iniciativa ${ }^{(2)}$.

Verifica-se, pelo quadro 1, que 19\% dos idosos sentiramse irritados/zangados no último mês. Sentimentos de medo, ansiedade, irritabilidade e mau humor são considerados sintomas do quadro de depressão nos idosos. As queixas de impaciência e o medo de possuir uma doença grave fazem parte do discurso dos idosos com essa sintomatologia ${ }^{(2)}$.

O sentimento de tristeza esteve presente em $42,8 \%$ dos idosos, $19 \%$ deles sentiram-se tristes por um período maior que poucas horas, e $4,8 \%$ sentiram-se tristes ou deprimidos no início do dia. Nota-se, ainda, que $42,8 \%$ dos idosos choraram ou sentiram vontade de chorar, e 14,3\% sentiram-se infelizes. Estudo realizado para identificar a presença de depressão em idosos residentes numa ILPI, mostrou que a tristeza foi o quinto sintoma mais referido pelos idosos, presente em $36,6 \%$ dos participantes ${ }^{(18)}$. Segundo essa investigação, a tristeza pode ocorrer em resposta à mudança provocada por um evento ou condição de uma pessoa querida, e às perdas constantes da normalidade.

O sentimento de tristeza pode estar relacionado ao tratamento despersonalizado e à perda da identidade e da individualidade, o que costuma ocorrer durante a institucionalização. Os idosos ficam desprovidos de autonomia e personalidade, o que pode acelerar o declínio de suas funções físicas e cognitivas, gerando tristeza e reduzindo-lhes o tempo de vida ${ }^{(19)}$.

Verifica-se, pela tabela 1, que $23,8 \%$ dos idosos apresentaram pensamentos suicidas e $28,6 \%$ sentiram que viver não valia a pena. As pessoas idosas não verbalizam a intenção de suicidar-se tão frequentemente quanto os adultos jovens, entretanto, é mais provável que tenham êxito ao tentar o suicídio. Isso se deve ao risco aumentado em idosos de cometer suicídio passivo, principalmente naqueles em que há quadro de depressão ${ }^{(4)}$.

Todas as ameaças de suicídio merecem atenção, deste modo, é importante que a Enfermagem reconheça não só as tentativas óbvias de suicídio, mas também aquelas que são sutis e igualmente destrutivas, como o uso inapropriado de medicamentos, a autoinanição e a prática de atividades contrárias às necessidades terapêuticas. O idoso suicida necessita de observação rigorosa, proteção cuidadosa e terapia imediata. O ambiente deve ser seguro e é necessário acompanhar esse idoso com disposição para ouvi-lo, de maneira que ele se sinta capaz de expressar seus pensamentos, a fim de impedir que leve a cabo o suicídio ${ }^{(20)}$.

Destaca-se, na tabela 1 , que $57,1 \%$ dos idosos não mencionaram expectativas para o futuro, e 9,6\% deles descreveram negativamente suas expectativas para $o$ futuro. Esse sentimento ou visão negativa sobre o futuro pode ser entendido também como desesperança. Um idoso desesperançoso vê pouca ou nenhuma alternativa/ escolhas que causem otimismo em relação ao futuro ${ }^{(4)}$.

A desesperança foi o sintoma depressivo mais frequente apontado em pesquisa desenvolvida com idosos institucionalizados $^{(18)}$. Esse sintoma não permite ao indivíduo perceber a disponibilidade de alternativas ou escolhas para a solução de problemas ou para a obtenção do que é desejado. A desesperança e a visão negativa sobre o futuro podem estar associadas às vivências do idoso depressivo, como a perda de um companheiro, de amigos, ou das capacidades funcionais ${ }^{(4)}$.

Observa-se, na tabela 1 , que $28,6 \%$ dos idosos sentiam que perderam o interesse ou a satisfação pelas coisas. A perda de interesse ou falta de motivação pelas atividades cotidianas de autocuidado podem estar presentes em idosos depressivos. Nesse sentido, os idosos física e cognitivamente capazes de realizar atividades diárias, mas que perdem o interesse pelo autocuidado e recusam-se a realizar essas atividades, podem estar deprimidos ${ }^{(4)}$.

\section{CONCLUSÕES}

Infere-se que o índice de sintomatologia depressiva verificado entre os idosos institucionalizados na ILPI em questão pode ser considerado elevado, uma vez que aproximadamente $43 \%$ da amostra apresentou indicadores de depressão. Entretanto, é necessário destacar que esse resultado indica a presença de sintomatologia depressiva, e não se refere ao diagnóstico de depressão; para tanto, seria necessária a utilização de escalas específicas e adequadas para esse fim, aplicadas por profissionais que possuam tal competência.

A desesperança emergiu como um elemento majoritário entre os idosos. Para esses, é impres-

Cogitare Enferm. 2011 Jul/Set; 16(3):418-23 
cindível um olhar atento para o estabelecimento de um plano de cuidados personalizado. Somente o cuidado específico e individualizado poderá garantir melhoras na capacidade de ter esperança destes idosos. Acredita-se que a ILPI só caminha, adequadamente, quando seus residentes possuem expectativas positivas quanto ao futuro; em outras palavras, quando os idosos possuem esperança.

Ressalta-se que a detecção precoce dos sintomas depressivos é importante para evitar o desenvolvimento do quadro depressivo, prevenindo, desta forma, seus efeitos negativos para a saúde e qualidade de vida desses idosos. Para tanto, é essencial a capacitação dos profissionais de enfermagem que atuam em instituição de longa permanência para idosos, ou seja, torná-los competentes para o olhar dirigido à sintomatologia secundária.

\section{REFERÊNCIAS}

1. Moraes EN. Princípios básicos de geriatria e gerontologia. Belo Horizonte: Coopmed; 2008.

2. Silva AS, Cunha UGV. Depressão: diagnóstico e tratamento. In: Moraes EN. Princípios básicos de geriatria e gerontologia. Belo Horizonte: Coopmed; 2008. p. 343-50.

3. Gordilho A. Depressão, ansiedade, outros distúrbios afetivos e suicídio. In: Freitas EV, Py L, Neri AL, Cancado FAX, Gorzoni ML, Rocha SM. Tratado de geriatria e gerontologia. Rio de Janeiro: Guanabara Koogan; 2002. p 204-15.

4. Roach S. Introdução à enfermagem gerontológica. Rio de Janeiro: Guanabara Koogan; 2003.

5. Blazer DG, Koenig HG. Transtornos do humor. In: Busse EW, Blazer DG. Psiquiatria geriátrica. $2^{\mathrm{a}}$ ed. Porto Alegre: Artmed; 1999. p. 245-72.

6. Bertolucci PHF, Brucki SMD, Campacci SR, Juliano Y. O miniexame do estado mental em uma população geral. Impacto da escolaridade. Arq Neuro-Psiquiatr. 1994;52(1):1-7.

7. Folstein MF, Folstein SE, Mchugh PR. Mini-mental state: a practical method for grading the cognitive status of patients for the clinician. J Psychiatr Res. 1975;12(3):189-98.

8. Brucki SMD, Nitrini R, Caramelli P, Bertolucci PHF, Okamoto IH. Sugestões para o uso do mini-exame do estado mental no Brasil. Arq Neuro-Psiquiatr.
2003;61(3B):777-81.

9. Veras RP, Silva SS. Questionário BOAS (Brazil OldAge Schedule) versão 2000. [internet]. [acesso em 21 de outubro de 2010] Disponível: http://www.unati.uerj.br/ boas/q_boas.htm

10. Veras RP. País jovem com cabelos brancos: a saúde do idoso no Brasil. Rio de Janeiro: Relume Dumará; 1994.

11. Siqueira GR, Vasconcelos DT, Duarte GC, Arruda IC, Costa JAS, Cardoso RO. Análise da sintomatologia depressiva nos moradores do abrigo Cristo Redentor através da aplicação da escala de depressão geriátrica (EDG). Cienc Saude Colet. 2009;14(1):253-9.

12. Cheloni CFP, Pinheiro FLS, Filho MC, Medeiros AL. Prevalência de depressão em idosos institucionalizados no município de Mossoró/RN segundo escala de depressão geriátrica (Yesavage). Expressão. 2003;34(1-2):61-73.

13. Plati MCF,Covre P, Lukasova K, Macedo EC. Depressive symptoms and cognitive performance of the elderly: relationship between institutionalization and activity programs. Rev Bras Psiquiatr. 2006; 28(2):118-21.

14. Valvanne J, Juva K,Erkinjuntti T, Tivis R. Major depression in the elderly: a population study in Helsinki. International Psychogeriatrics. 1996;8(3):437-43.

15. Lenardt MH, Michel T, Tallmann AEC. A condição de saúde de idosos residentes em instituição de longa permanência. Cogitare Enferm. 2009;14(2):227-36.

16. Fortes-Burgos ACG, Neri AL, Cupertino APFB. Eventos estressantes, estratégia de enfrentamento, autoeficácia e sintomas depressivos entre idosos residentes na comunidade. Psicol Refl e Crit. 2008;21(1):74-82.

17. Xavier FMF, Ferraz MPT, Bertollucci P, Poyares D, Moriguchi EM. Episódio depressivo maior, prevalência e impacto sobre qualidade de vida, sono e cognição em octogenários. Rev Bras Psiquiatr. 2001;23(2):62-70.

18. Andrade ACA, Lima FRA, Silva LFA, Santos SSC. Depressão em idosos em uma instituição de longa permanência (ILP): proposta de ação de enfermagem. Rev Gaúch Enferm. 2005;26(1):57-66.

19. Lenardt MH, Michel T, Wachholz PA. Autoavaliação da saúde e satisfação com a vida de idosas institucionalizadas. Ciênc Cuid Saúde. 2010;9(2):246-54.

20. Eliopoulos C. Enfermagem gerontológica. $5^{\mathrm{a}}$ ed. Porto Alegre: Artmed; 2005. 